\title{
Madeleine Leininger: un análisis de sus fundamentos teóricos
}

Lic. Olivia Aguilar Guzmán*, Lic. Miroslava Iliana Carrasco González*, Lic. María Aurora García Piña*, Lic. Araceli Saldivar Flores*, y Mtra. Rosa María Ostiguín Meléndez **

*Alumnas de la 3a. Generación de la Maestría en Enfermería de la ENEO .2006.

** Tutor del Programa de Maestría en Enfermería ENEO -UNAM.

El presente artículo tiene como propósito hacer un análisis de los fundamentos teóricos de la propuesta de Madeleine Leininger, que se sustenta en dos disciplinas; Antropología y Sociología. El trabajo contiene los antecedentes biográficos de la autora, planteamientos de la antropología filosófica, social y cultural, a partir de los cuales se hacen inferencias en relación a los constructos utilizados por Leininger. Finalmente se establece que la Enfermería requiere del conocimiento del ser humano que vive dentro de una estructura social donde la cultura determina el estado de bienestar/ salud.

PALABRAS CLAVE: sustentos teóricos, cuidados culturales y Enfermería.

\section{Abstract}

The present article has like intention make an analysis of the theoretical foundations of the proposal of Madeleine Leininger, that is sustained in two disciplines; Anthropology and Sociology. The work contains the biographical antecedents of the author, expositions of the philosophical, social and cultural anthropology, from which inferences in relation to the constructos used by Leininger become. Finally one settles down that the nursing requires of the knowledge of the human being who lives within a social structure where the culture determines the well-being state health.

KEY WORDS: theoretical sustenances, cultural cares and nursing.

\section{BIOGRAFÍA DE MADELEINE LEININGER}

Madeleine Leininger nació en Sutton, Nebraska, inició su carrera profesional después de diplomarse en la escuela de Enfermería de St. Anthony, en Denver. En 1950, obtuvo el título de Ciencias Básicas en Biología, (Kansas) y realizó estudios complementarios de Filosofía y Humanismo. Para 1954, obtuvo una Maestría en Ciencias de Enfermería Psiquiátrica, por la Universidad Católica de América en Washington D.C.En 1955 durante su ejercicio profesional, en el área de Psiquiatría Infantil observó que el personal sanitario no conocía suficientemente los factores culturales que influían para su asistencia; a partir de esta experiencia nace la inquietud de construir un modelo que considere una visión transcultural del cuidado para el individuo, idea que comparte con Margarett Mead (Antropóloga)’.
Durante los 60's, aplicó e impulso el empleo de métodos cualitativos de investigación; coordinó el primer curso sobre Enfermería Transcultural en la Universidad de Colorado; fundó el Committee on Nursing and Anthropology y dirigió los programas de Doctorado en ésta misma área. Para 1974 en la Universidad de UTAH, en Salt Lake City, fue pionera del programa de master y doctorado; fundó la Sociedad Nacional de Enfermería Transcultural y posteriormente en 1989, el Journal of Transcultural Nursing del que fue editora².

En los últimos años trabajó en áreas de investigación y consultoría de Enfermería ${ }^{3}$, hasta el 2006 en que fallece. Sin embargo, la Teoría ha permanecido vigente gracias a 
las investigaciones que se realizan actualmente ante las necesidades culturales del mundo.

\section{SUSTENTOS TEÓRICOS DE LEININGER}

A partir de la revisión de la Teoría de "Cuidados Culturales: Teoría de la Diversidad y Universalidad", se identifica la influencia de dos disciplinas para su construcción: Ia Antropología y la Sociología mismas que permiten visualizar al individuo en su contexto sociocultural (Ver cuadro 1).

Como se puede ver, de la primer disciplina se deriva la Antropología Filosófica ${ }^{4,5}$ en donde se define ¿Qué es el hombre? desde una visión ontológica, como dice Aristóteles "el alma y el cuerpo constituyen el compuesto humano, el cual tiene diversas actividades: vegetativas, sensitivas y racionales; en cambio Kant refiere al hombre concreto y sensible; las críticas que hacen tratan del yo y especialmente del hombre moral ubicado por encima de la sensibilidad y se puede resumir así: 1. ¿Qué puedo saber?, 2. ¿Qué debo hacer?, 3. ¿Qué me cabe esperar? y 4. ¿Qué es el hombre? La respuesta a estas interrogantes se encuentran respectivamente en la metafísica, la moral, la religión y la Antropología. Por su parte Hegel hace referencia a lo humano, para comprender la filosofía del espíritu que se desarrolla en tres grandes etapas dia lécticas: espíritu objetivo, subjetivo y absoluto ${ }^{6}$. Bajo ésta óptica Fawcett, afirma que se "utilizan conocimientos de la
Antropología para descubrir las dimensiones Ontológicas y Epistemológicas del bienestar y la salud"8.

\section{Definición de persona}

Leininger define a la persona como: "ser humano, que cuida, ser cultural, familia, grupo, comunidad, institución social, cultura y subcultura". Por otro lado, se refiere al "bienestar-salud" desde un punto de vista holístico. Término que se considera necesario revisar a partir de dos posturas psicológicas: La Escuela de la Gestalt y de Kurt Goldstein ${ }^{9}$; ambos enfoques permiten visualizar al individuo como un ente biopsicosocial.

Desde ésta concepción de hombre, la Antropología social y cultural describe y analiza la influencia de la cultura inmersa en un contexto determinado, representada por cuatro corrientes de pensamiento: Funcionalismo, Estructuralismo, Particularismo Histórico y Marxismo Estructural; que aportan elementos en donde el individuo se desarrolla como parte de una estructura social, como a continuación se describe.

\section{Análisis del contexto ambiental}

El Funcionalismo, estudia las culturas a partir de cómo se organizan para satisfacer las necesidades de un grupo humano, es decir, todas aquellas tareas u objetivos que

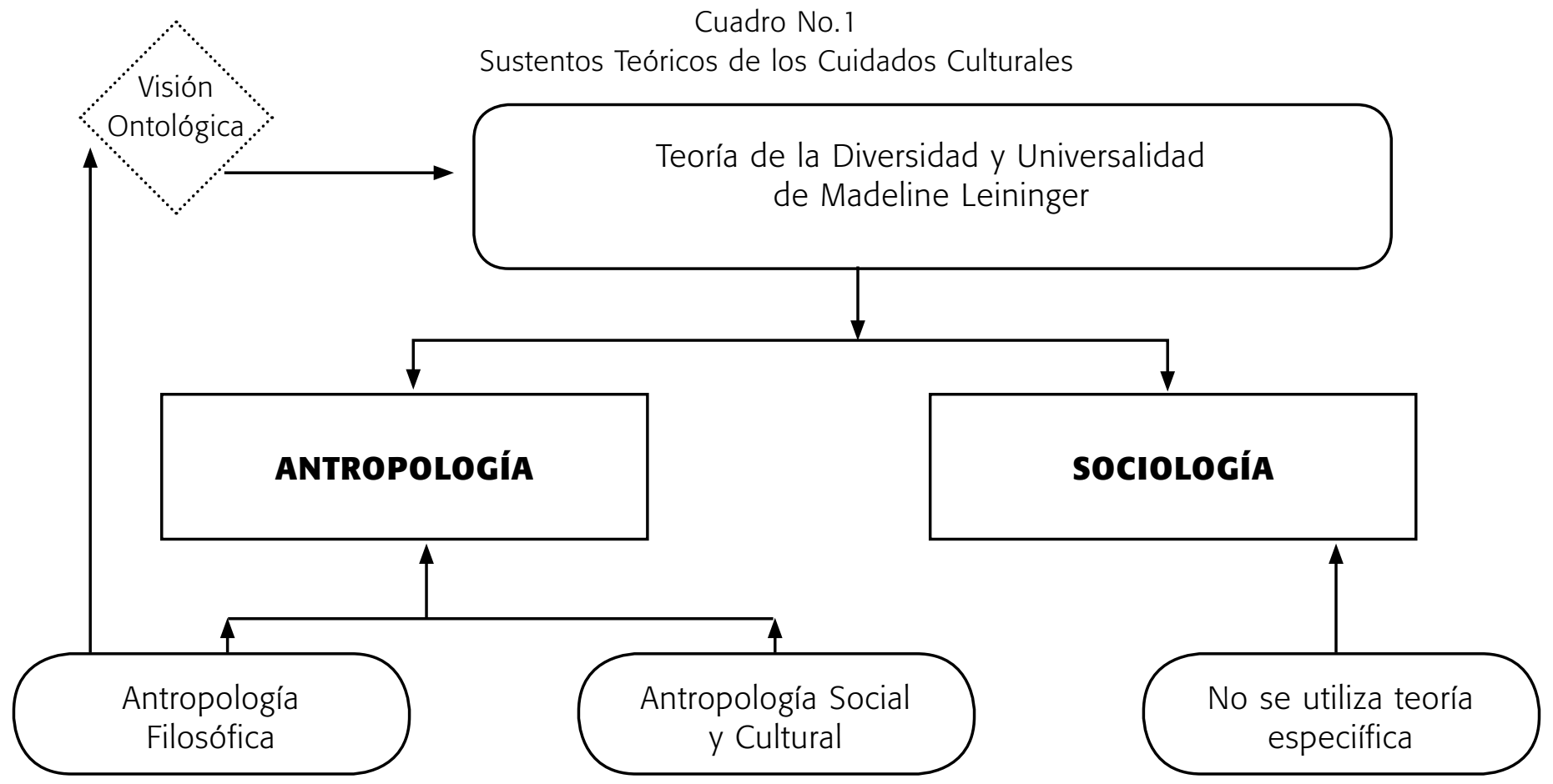


tienden a mantener y conservar los organismos de la sociedad, incluyendo sus modelos culturales, a partir de esto es probable que Leininger eligiera elementos como: visión del mundo, estructura social, comunidades y sociedades para construir su teoría.

Con estos planteamientos se inicia el estudio de las culturas de los pueblos por medio de la Etnografía* y Etnología*; donde se visualizan los estratos sociales y sus interrelaciones, dando origen al Estructuralismo francés que se enfoca en las ciencias humanas para analizar el lenguaje, la cultura y la sociedad ${ }^{10}$. De acuerdo con ésta teoría, dentro de una cultura el significado se produce y reproduce a través de varias prácticas como la preparación de la comida y los rituales para servirla, ritos religiosos, juegos, textos literarios y otras formas de entretenimiento. Podría pensarse que Leininger construye conceptos de dimensiones estructurales, culturales-sociales, factores religiosos y filosóficos; grupo-sociedad, contextos ambientales, lenguaje, etnohistoria, sistemas genéricos tradicionales, así como remodelación de los cuidados culturales. Al observar la diversidad cultural surge la necesidad de estudiar a cada cultura por separado lo que da origen al Particularismo Histórico, en donde se reconoce que no existe una cultura general o global y la influencia del determinismo geográfico frente a los factores culturales, por otro lado el relativismo cultural diversifica al individuo y obstaculiza su estudio frente al resto de pueblos y culturas. Con ésta misma ideología Margaret Mead trabajó en la antropología y estudió en diferentes culturas, la crianza y educación en niños; con ella se inicia el interés en el estudio de la infancia y la mujer; específicamente la concepción holistica de la cultura.

Con lo expuesto, puede pensarse que Leininger retoma de éstas ideas los aspectos de: diversidad cultural, cultura, etnografía, valores culturales, estilos de vida, individuo, familia, grupos, comunidad, relativismo cultural, preservación del cuidado, etnocentrismo, influencias, patrones, expresiones, actividades de asistencia y educación.

Por otra parte, Leslie White y Julian Steward influenciados por Marx y Engels inician el Marxismo Estructural, el cual sostiene que la esencia humana no es algo abstracto inherente a cada individuo. Es en su realidad, el conjunto de sus relaciones sociales, establecidas a través de la interacción del hombre con la naturaleza en el proceso de transformación de su vida material y espiritual. A partir de ésta corriente de pensamiento se infiere que Leininger utiliza los siguientes conceptos: cultura, diversidad cultural, dimensiones estructurales, sociedad, grupos, bienestar, biotecnología, factores legales y políticos.
Lo anterior confirma la primera definición que Leininger propone del contexto ambiental, que se describe como "la totalidad de un evento, situación o las experiencias particulares que dan el significado a las expresiones humanas, interpretaciones e interacciones sociales, específicamente las escenas físicas, ecológicas, sociopolíticas y/o culturales; en otras palabras "la totalidad de un evento, situación y experiencia de vida da significado y guía las expresiones humanas, así como decisiones dentro de un área geográfica particular"7.

\section{Diversidad cultural}

Cabe mencionar que la Teoría de Leininger no emplea ninguna teoría Sociológica específica que la sustente, aunque si considera algunos conceptos relevantes de ésta disciplina a través de las redes sociales de las familias; es decir a través de los escenarios de las relaciones humanas con sus regularidades, contradicciones y conflictos los cuales configuran patrones de comportamiento colectivo que explican la particularidad de una sociedad determinada, de esta manera se estudia la diversidad social, en este sentido la sociedad está formada por individuos que actúan colectivamente por medio de las costumbres, educación, sexo, edad, ocupación o las creencias en una causa determinada. Es Weber, quien describe como comprender ésta vida colectiva, a través de la acción social, en concreto cuando actuamos socialmente estamos suponiendo una respuesta por parte de otros individuos.

La teoría Marxista sostiene que por encima de las relaciones económicas se establecen otras de origen ideológico a lo que llama superestructura de las clases sociales de distintos grupos. Ahora bien hablar de cultura alude a las formas de nacer y morir, de casarse y de comer, a los temores, los tabúes y los mitos, a las formas de interacción social, de conducta, de solidaridad social, de conciencia pero también a los patrones de reproducción y de organización social y política, además de las ideas religiosas y morales, las leyes, las tradiciones y las costumbres. En concreto, el concepto de cultura se refiere a los valores que los miembros de un grupo social dado comparten, a las normas que acatan y a los bienes materiales que crean. En particular los valores son ideales abstractos que varían de una sociedad a otra, mientras que las normas son principios o reglas que se espera observen los miembros de una sociedad ${ }^{10}$. Lo anterior se concreta con el concepto de cultura propuesto por Leininger: "Conjunto de valores, creencias, normas y estilos de vida aprendidos, compartidos y transmitidos dentro de un grupo que orientan sus razonamientos, decisiones y acciones, según esquemas determinados" ${ }^{11}$. 


\section{APORTACIONES PRINCIPALES DE LEININGER}

De todo lo anterior, podemos analizar que algunos de los principales conceptos de la teoría de Leininger son: "cuidado, atención, cultura, valores culturales y diferencias culturales; especificó que la atención era el tema central del cuidado, el conocimiento y la práctica Enfermera, puesto que la atención incluye los actos para ayudar y dar soporte o facilidades a individuos o grupos con necesidades evidentes o previsibles, también sirve para mejorar las condiciones humanas y los modos de vida"12.

La teoría transcultural se puede visualizar a través del "modelo del sol naciente" el cual describe al ser humano como un ente integral, que no puede separarse de su procedencia cultural ni de su estructura social, de su concepción del mundo, lo que constituye uno de los principios fundamentales de la teoría de Leininger, él cual se divide en cuatro niveles":

Estos niveles van de más a menos abstracto y los tres primeros niveles proporcionan conocimientos para brindar cuidados culturalmente coherentes. En el nivel uno se representa la visión del mundo y los sistemas sociales, permite el estudio de la naturaleza, el significado y los atributos de los cuidados desde tres perspectivas: microperspectiva (los individuos de una cultura), perspectiva media (factores más complejos de una cultura especifica) y macro perspectiva (fenómenos transversales en diversas culturas). Estas características representan el entorno donde se desenvuelve el individuo a través de estructuras sociales que define la Sociología.

En el nivel dos proporciona información acerca de los individuos, las familias, los grupos e instituciones en diferentes sistemas de salud pero también proporciona información acerca de los significados y expresiones específicas relacionadas con los cuidados de salud; lo cual es abordado por la Antropología con su método etnográfico el cual retoma Leininger para proponer la Etnoenfermería como herramienta para la recolección de información.

En el nivel tres, proporciona información acerca de los sistemas genéricos o tradicionales y profesionales, incluyendo la Enfermería, que actúan dentro de una cultura e identificación de la diversidad y universalidad de los cuidados culturales; se puede señalar que este nivel retoma planteamientos filosóficos del ser humano; por lo que es necesario rescatar y promover el trabajo multidisciplinario que permita a los profesionales brindar un cuidado integral a la persona tomando como referencia los conocimientos y habilidades tradicionales.

Finalmente el nivel cuatro determina el nivel de las acciones y decisiones de los cuidados Enfermeros; en esté nivel se proporcionan los cuidados Enfermeros que incluyen la preservación, acomodación y remodelación de los cuidados culturales (Cuidados culturalmente congruentes) ${ }^{13}$. De esta manera Leininger plantea visualizar al individuo de forma holística, siendo aquí determinante el factor cultural como eje central del modelo. Desde esta perspectiva de Enfermería, el reto es descubrir el significado de la conducta, flexibilidad, creatividad y conocimiento de las diferentes culturas para adaptar las intervenciones de Enfermería.

Lo anterior se constata con las publicaciones de investigaciones con enfoque cualitativo, que han utilizado el modelo de Leininger dando respuesta al bienestar de los grupos, familias y comunidades en diferentes contextos a nivel mundial.

\section{CONCLUSIONES}

Una vez realizado el análisis de los fundamentos teóricos de la propuesta de Madeleine Leininger sobre los cuidados culturales se concluye que existe una influencia antropológica que permite visualizar al hombre como un ser integral que vive dentro de una estructura social y cultural misma que determina el estado de bienestar / salud; él cual debe ser considerado por el profesional de Enfermería al proporcionar cuidados culturalmente congruentes.

Al finalizar este trabajo se descubre que es necesario que el profesional o estudiante de Enfermería profundice sobre el contexto histórico - social así como las aportaciones de las diferentes disciplinas en el cual se construyen las teorías, para comprender sus planteamientos y aplicabilidad dentro de la Enfermería.

Es importante la promoción y difusión del cuerpo conceptual de esta teoría para propiciar una sensibilización hacia otras formas de ver la salud y sus cuidados, permitiéndonos mediar los cuidados, principalmente a favor de poblaciones indígenas. Así, aplicar la teoría de la diversidad y universalidad en cualquiera de los ámbitos de la disciplina, práctica, educación e investigación requiere de especialización.

De hecho, la realización de éste ensayo en el programa de maestría favorece sin duda el desarrollo de habilidades de pensamiento crítico y de investigación documental. 


\section{RECOMENDACIONES}

- Explorar los elementos sociológicos utilizados en la construcción de la teoría transcultural de Leininger.

- Realizar investigaciones de Enfermería con enfoque transcultural utilizando los constructos propuestos por Leinger.

- Realizar instrumentos de valoración de Enfermería con base a los conceptos básicos de M. Leininger, con la finalidad de favorecer el cuidado transcultural y por ende no solo atender a las personas con enfoque biologicista.

- Esta teoría permite retomar el trabajo de la Enfermera profesional para brindar cuidado en la atención Comunitaria para el individuo y la familia sana o Enferma.

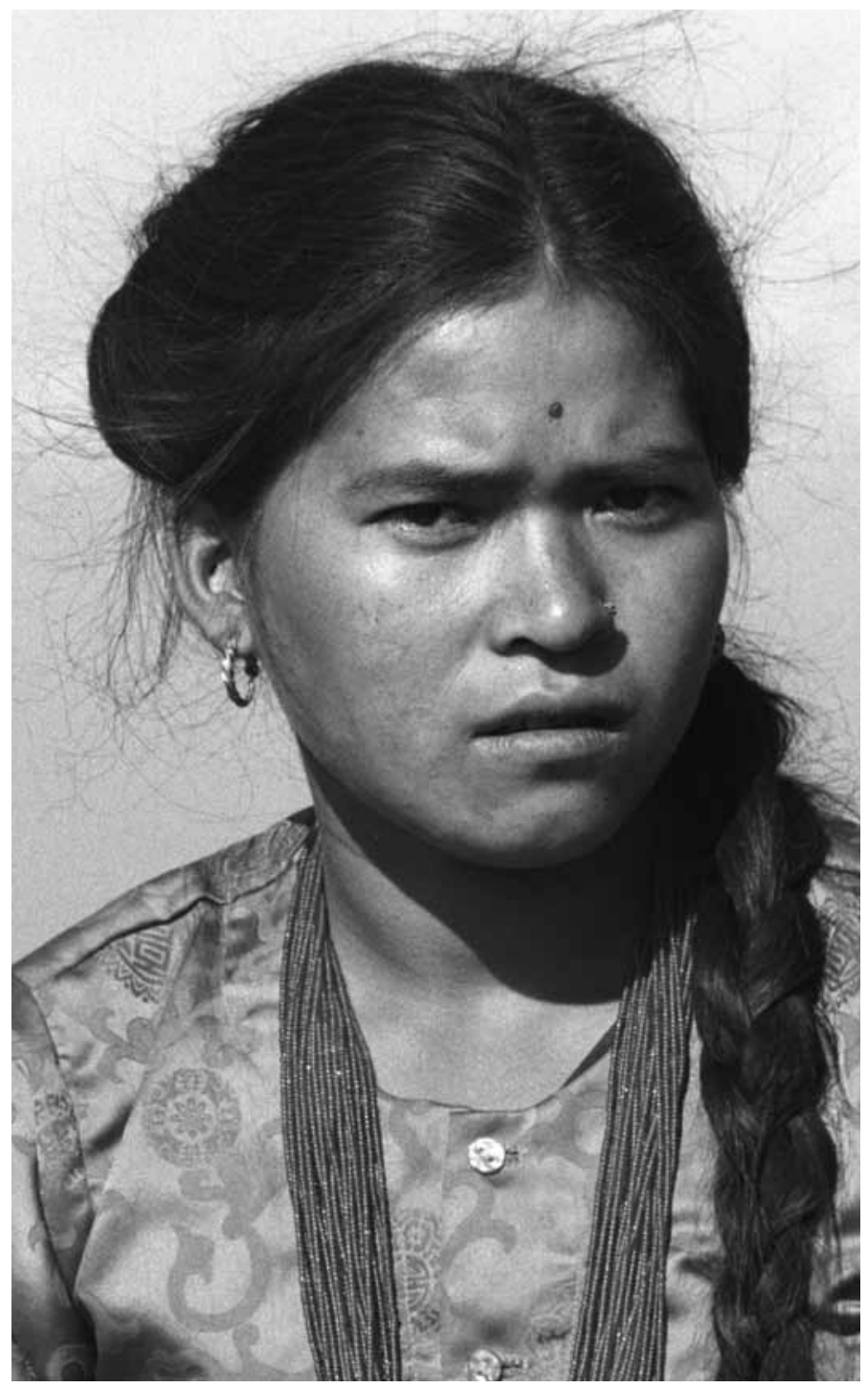

\section{NOTAS}

**Etnología: Es la ciencia social que estudia y compara los diferentes pueblos del mundo, busca establecer relaciones comparativas entre las características de los diferentes pueblos humanos, aspectos como: diversidad cultural, parentesco, sus influencias, subsistencia, sistemas económicos de las culturas, civilizaciones, religión, expresión simbólica trascendental, organización familiar, sistemas sociales y políticas.

* Etnografía: Del griego Ethnos (tribu, pueblo), grapho yo escribo; literalmente descripción de las culturas de los pueblos existentes. Es un método para realizar investigación cualitativa.

\section{REFERENCIAS BIBLIOGRÁFICAS}

1 Maja, T. Ledimensioni culturali della professione infermieristica., Savar Rivista del nursing in movimento Italia, 2005 (3):3-14.

2 Marriner T, A. Cuidados Culturales: Teoría de la diversidad y universalidad. En: Modelos y Teorías de Enfermería, $4^{\mathrm{a}}$ ed. Ed. Mosby. Madrid, 1999. p. 439-442.

3 Oriá, MOB; Ximenes, LB; Alves, MDS. Madelein Leininger and the teohry of the Cultural Care Diversity and Universality: an Historical Overview. Online Braz J Nurs. 2005 August; 4(2).

4 Beals L, R; Hoijer, H. Introducción a la Antropología. Ed. Aguilar. New York. 1981. p. 3-22

5 Sánchez O, S. Texto Básico de Autoenseñanza. 2ª . ed..UNAMENEO-SUA. 2000. p. 137

6 Buber, M. ¿Qué es el hombre? Fondo de Cultura Económica. México, 1992. p.11-54.

7 Fawcett, J. Análisis and Evaluation of Contemporary Nursing Knowledge. Nursing Models and Theories. Ed. F.A. Davis Company. Philadelphia. 2000. p. 51 1- 547

8 Sahakian W, S. Historia de la Psicología, $2^{a}$ ed. Edit. Trillas México, 1990. p.473-491.

9 Puga, C; Peschard, J; Castro, T. Hacia la sociología. 3a. Ed. Pearson, México, 1999. p.63-69, 120.

10 Ibid. p. 13-62.

11 Fawcett, J. Op.cit. p. 517.

12 Marriner T; A. Cuidados Culturales: Teoría de la diversidad y universalidad en: Modelos y Teorías de Enfermería, $5^{a}$ ed. Ed. Mosby. Madrid, 2005. p. 501-527.

13 Wesley, R L. Teorías y Modelos de Enfermería. $2^{a}$ ed. Ed. McGraw-Hill Interamericana, México.1997. p.119-125.

\section{DIRECCIÓN PARA CORRESPONDENCIA}

Lic. Olivia Aguilar Guzmán: ougo690418@yahoo.com.mx

Lic. Miroslava Iliana Carrasco González: sorimi15@hotmail.com.mx

Lic. María Aurora García Piña: augarpi@yahoo.com.mx

Lic. Araceli Saldívar Flores: arasal21@ yahoo.com.mx 